\title{
A simple and effective method for addition silicone impression disinfection
}

\author{
Maria João Azevedo ${ }^{1}$, Inês Correia ${ }^{1}$, Ana Portela ${ }^{1 *}$, Benedita Sampaio-Maia ${ }^{1,2,3}$ \\ ${ }^{1}$ Faculty of Dental Medicine, Universidade do Porto, Porto, Portugal \\ ${ }^{2}$ National Institute of Biomedical Engineering, Universidade do Porto, Porto, Portugal \\ ${ }^{3}$ Institute of Research and Innovation in Health, Universidade do Porto, Porto, Portugal
}

\begin{abstract}
PURPOSE. Although dental impression disinfection is determinant to reduce the cross-infection risk, some studies have shown that, in real practice, the disinfection procedures vary considerably. Thus, the aim of this study was to evaluate the antimicrobial effectiveness and the impact on the dimensional stability of addition silicone' impressions of water wash and the most clinically used disinfection solutions: $3 \%$ hydrogen peroxide, commercial disinfectant MD520 (Durr) and 1\% and 5.25\% sodium hypochlorite. MATERIALS AND METHODS. For this investigation, dental impressions were taken on 16 volunteer dental students. The antimicrobial effectiveness of each procedure was evaluated by pour plate method. The dimensional stability was evaluated using a standardized stainless-steel model, according to ANSI/ADA nº19 specification. RESULTS. The study results showed that water wash does not alter the dimensional stability of addition silicone impressions but doesn't reduce the microbial load of the material $(P>.05)$. On the other hand, addition silicone disinfection by immersion with 3\% hydrogen peroxide, MD520 (Durr), or sodium hypochlorite at 1\% and 5.25\% does not alter the dimensional stability significantly but reduces $>99.9 \%$ of the microbial load of the impressions $(P<.001)$. CONCLUSION. Addition silicone impressions should always be disinfected after water wash in order to reduce effectively the cross-infection risk. All disinfectants tested showed high antimicrobial efficiency without significant changes in three-dimensional shape of impressions. Hydrogen peroxide and sodium hypochlorite are of particular importance because are easily accessible in dental setting. The less explored hydrogen peroxide could be a valuable alternative for silicone impressions disinfection. [J Adv Prosthodont 2019;11:155-61]
\end{abstract}

KEYWORDS: Silicone elastomers; Disinfection; Sodium hypochlorite; Hydrogen peroxide

\section{INTRODUCTION}

Cross-infection is a relevant matter in dental medicine practice. ${ }^{1-3}$ Dental impressions are inevitably contaminated by contact with saliva, blood and bacterial plaque, which contains potentially pathogenic microorganisms such as Streptococcus,

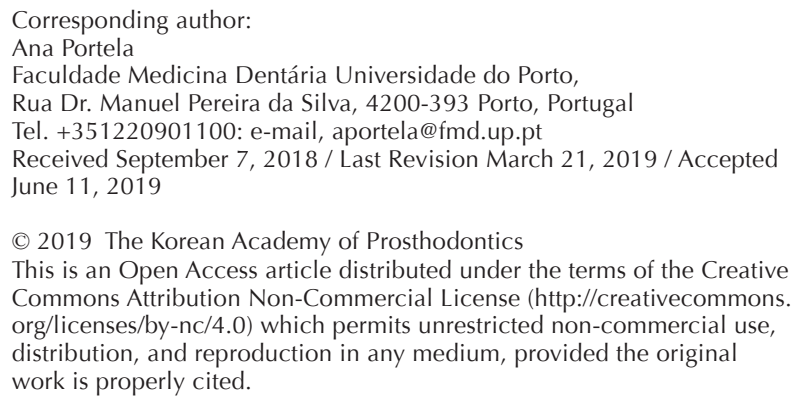

This work was supported by Faculty of Dental Medicine of University of Porto.
Staphylococcus, Escherichia coli, Mycobacterium tuberculosis, in addition to hepatitis $\mathrm{B}$ and $\mathrm{C}$ viruses, human immunodeficiency virus (HIV) and herpes simplex virus. ${ }^{4-7}$ Dentists, dental assistants, and prosthetist laboratory technicians are all at risk of cross-infection., ${ }^{8,9}$

Up to 1991, washing of dental impressions under tap water was the recommended procedure for their disinfection. However, this method only partial eliminates the bacterial, viral, and fungal load, not avoiding the risk of potential infection. ${ }^{1,6}$ Currently, the Australian Dental Association and the Center for Disease Control (CDC) recommend disinfecting dental impressions immediately after their removal from the mouth. ${ }^{10,11}$ Moreover, impressions disinfection through immersion, in opposition to spray, is considered the safest procedure, since all surfaces are equally covered by disinfectant. ${ }^{12}$ Impressions disinfected by immersion should be time-limited and the Australian Dental Association recommends a maximum period of 15 minutes. ${ }^{10}$ However, there is still some reluctance in disinfecting dental impres- 
sions due to the possible degradation of the impression quality and the resulting cast. ${ }^{13}$ Some studies reveal that in real practice impressions' disinfection may vary significantly in different laboratories or even different countries. ${ }^{12,14}$ In this scenario, exploring a simple, accessible but efficient impression disinfection method is of major relevance.

A disinfectant is a broad-spectrum chemical agent that may have bacteriostatic or bactericidal action and is used on an inanimate surface or object. ${ }^{15}$ The disinfectant selection depends on the inanimate surface or object to be use. From a wide variety of disinfectant solutions on the market, MD520 commercial disinfectant is a combination of aldehydes, quaternary ammonium compounds, special surfactants and adjuvants in aqueous solution, having a bactericidal, tuberculocidal, and virucidal action (virus with and without envelope). ${ }^{16}$ It has shown good results in some studies regarding the evaluation of dimensional stability of dental impressions ${ }^{17}$ and its antimicrobial efficacy. ${ }^{16,18}$ Besides its advantages, MD520 is not always available in dental settings.

Sodium hypochlorite and hydrogen peroxide are two low-cost effective disinfectants always present in dental office. Sodium hypochlorite is a water-soluble disinfectant used in surface and water disinfection. When dissolved in water, it forms hypochlorous acid, which, in turn, dissociates into hydrochloric acid and oxygen atoms, having a potent oxidizing effect. ${ }^{6}$ The antimicrobial action of sodium hypochlorite is fast, effective, and broad spectrum, according to the American Dental Association (ADA) protocol. ${ }^{18}$ Hydrogen peroxide is a well-known and used disinfectant and its effects are caused by its oxidative action, potentiated when the hydrogen peroxide is in the gas phase. ${ }^{2,19}$ Hydrogen peroxide represent traditional disinfectants of removable dentures. ${ }^{19}$ At concentrations of $3 \%$ and $5 \%$, it effectively reduces the biofilm of implant surfaces, periimplant tissue, and cutaneous wounds. ${ }^{20}$ It has a broad spectrum of action, which encompasses bacterial spores, bacte- ria, viruses, and fungi. ${ }^{4}$

Addition silicone is a synthetic elastomeric impression material launched in 1975. Since then, it has become the chosen impression material for many clinical situations, such as fixed prosthodontics, conservative dentistry, implantology, and removable prosthodontics, due to its excellent physical properties and handling characteristics. ${ }^{21,22}$ Therefore, taking into account its clinical uses, disinfection should not affect the dimensional stability of silicone dental impression, which is defined as the ability of a material to maintain its size and three-dimensional shape. This is an essential requirement in dental practice in order to obtain accurate replicas of models and prostheses made from the models. ${ }^{23}$

Thus, a disinfectant for impression materials should ideally fulfill two fundamental requirements; it must be an effective antimicrobial agent and must preserve the dimensional stability and the surface details of both the impression and the resulting cast to achieve a greater clinical accuracy. ${ }^{3,24}$ Therefore, the objective of this study was to explore a simple but efficient method for dental impression disinfection using low-cost and easily accessible disinfectants. With this aim, we evaluated the antimicrobial effectiveness and the impact on dimensional stability of addition silicone' impressions of the most clinically used disinfection solutions: $1 \%$ and $5.25 \%$ sodium hypochlorite, $3 \%$ hydrogen peroxide, and a commercial disinfectant MD520 (Durr), and compare it with no washing or water washing.

\section{MATERIALS AND METHODS}

For this investigation, the selected impression material was addition silicone (Table 1). The chosen disinfectant solutions were 3\% hydrogen peroxide (Scharlau, Barcelona, Spain), commercial MD520 disinfectant (Durr, BietigheimBissingen, Germany), as well as $1 \%$ and $5.25 \%$ sodium hypochlorite (Limpolar, Fiães, Portugal) (Table 2).

Table 1. Handling instructions of the impression material Addition Silicone-Hydrorise Putty (at $35^{\circ} \mathrm{C}$ )

\begin{tabular}{cccccc}
\hline Use & Type of Setting & $\begin{array}{c}\text { Working time } \\
(\text { min }: s)\end{array}$ & $\begin{array}{c}\text { Time in mouth } \\
\text { (min :s) }\end{array}$ & $\begin{array}{c}\text { Time of setting } \\
\text { (min : s) }\end{array}$ & $\begin{array}{c}\text { Brand name / } \\
\text { Supplier }\end{array}$ \\
\hline Prostheses and Implantology & Fast & $1: 30$ & $2: 30$ & $4: 00$ & Zermack/Germany \\
\hline
\end{tabular}

Table 2. Description of the study groups

\begin{tabular}{|c|c|c|c|c|c|}
\hline Group & Treatment / Disinfectant & Concentration & Immersion time & Number of samples (n) & Brand name / Country \\
\hline 1 & No treatment (Control) & - & - & 10 & - \\
\hline 2 & Water wash & - & 30 seconds & 10 & - \\
\hline 3 & Hydrogen peroxide & $3 \%$ & 10 minutes & 10 & Scharlau / Spain \\
\hline 4 & MD520 & - & 5 minutes & 10 & Durr / Germany \\
\hline 5 & Sodium hypochlorite & $1 \%$ & 10 minutes & 10 & Limpolar / Portugal \\
\hline 6 & Sodium hypochlorite & $5.25 \%$ & 10 minutes & 10 & Limpolar / Portugal \\
\hline
\end{tabular}


The samples were divided into groups according to the disinfection protocol to which they had been subjected (Table 2). There was a control group (not washed) and a group washed with cold tap water for 30 seconds. For all disinfectants (groups 3 to 6), the impression was first rinsed for 15 seconds under tap water, was then immersed in disinfectant during a specific time (Table 2), and again rinsed for 15 seconds under tap water.

For the evaluation of antimicrobial efficiency, dental impressions were taken on 16 students of the Faculty of Dental Medicine of the University of Porto, who volunteer for this study. The inclusion criteria were the absence of systemic and salivary pathologies, age between 22 and 25 years old, and a decay, missing, filled teeth (DMFT) index less than 5 . The study protocol was approved by the Ethics Committee of Faculty of Dental Medicine of the University of Porto and all recruited students were asked to give their free written informed consent. For each volunteer, a partial impression in the area of the $1^{\text {st }}$ and $2^{\text {nd }}$ lower left or right molars was taken with addition silicone, under aseptic conditions. These impressions were divided into 6 samples of equal size. Each part was washed / disinfected following one of the protocols (groups 1 to 6). For comparison, an impression on sterile artificial teeth (Frasaco GmbH, Tettnang, Germany) was also taken to evaluate the microbial load of the impression materials (basal) $(n=6)$. After disinfection, the samples from groups 3 to 6 (disinfected) were placed in a bead tube with $3 \mathrm{~mL}$ physiological serum $(0.9 \% \mathrm{NaCl})$ and vortexed 5 seconds for 3 times. Then, $1 \mathrm{~mL}$ of the solution was inoculated in triplicate by incorporation into a plate with the Brain Heart Infusion Agar medium (Liofilchem, Teramo, Italy). For samples not washed or washed with water (groups 1 and 2), a serial dilution up to 1:100 was performed, and seeded in triplicate in BHI. The basal (impression with Frasaco) was also inoculated by incorporation. All plates were incubated at $37^{\circ} \mathrm{C}$, aerobically, for 72 hours. After the incubation time, the Colony Forming Units (CFU's) were counted.

The dimensional stability was evaluated in an impression from a standardized stainless steel model, according to ISO 4823:2015, ANSI/ADA no19 specification for elastomeric impression materials. ${ }^{25,26}$ The model has 3 parallel horizontal lines that cross its surface, with $25000 \mu \mathrm{m}$ long (side A), and 2 shorter vertical lines, with $5000 \mu \mathrm{m}$ long (side B), at each end (Fig. 1). ${ }^{3}$ Before taking the impression, the model was washed for 20 seconds with $70 \%$ ethanol and placed $1 \mathrm{~min}$ ute in the greenhouse at $37^{\circ} \mathrm{C}$ to simulate the temperature of the oral cavity. Then, the impression material samples were handled according to the manufacturer's instructions (Table 1) for the stipulated time. After the impression material had been set, it was removed from the model and submitted to the protocol of one of the test groups described above (group 1 to 6 ). Afterwards, the impressions were visualized with a magnifier glass (Wild / Leica M420) where, under a magnification of 7.9X, they were photographed and saved for later analyses with the Image J $1.51 \mathrm{j} 8$ software. Measurements were made 3 times for each of the $60 \mathrm{sam}$ -

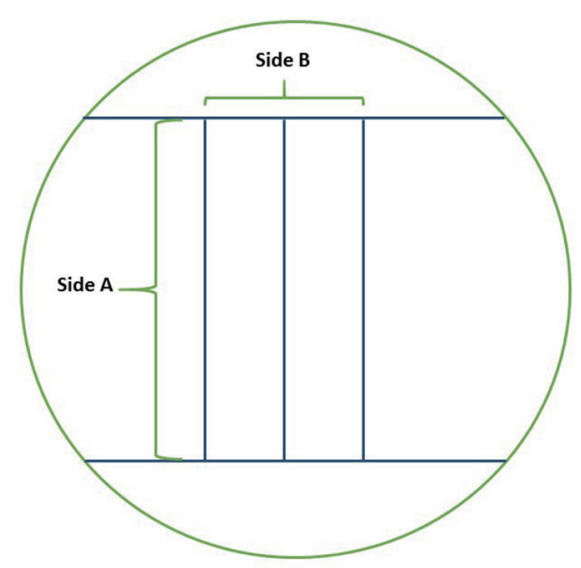

Fig. 1. Scheme of the standard model surface and geographic reference to side $A$, which consists on 3 parallel lines with $25000 \mu \mathrm{m}$ long, and side B, represented by 2 parallel lines with $5000 \mu \mathrm{m}$ long.

ples, on both sides A and B.

For statistical analysis, the software IBM SPSS 24 software (Statistical Package for Social Sciences) was used. Normality was assessed with Shapiro-Wilk test. Results were analyzed using ANOVA test for normal distribution or Kruskal-Wallis for non-normal distribution. When assumption of homogeneity of variances was violated for ANOVA, Brown and Forsythe test was applied. The minimum level of significance $(\alpha)$ applied was $5 \%$, with the Bonferroni correction for multiple comparisons. Values for antimicrobial efficiency were expressed as $\%$ of control. The power of the analysis was calculated. Values for dimensional stability were expressed as dimensional changes, calculated as the differences between the measurements obtained from the impressions and the real value of the standardized model (25000 or $5000 \mu \mathrm{m})$.

As for the statistical hypothesis regarding the assessment of the dimensional stability, we established: $\mathrm{H} 0=$ there are no significant differences in the dimensional stability of the impressions after water washing or disinfecting the impressions; $\mathrm{H} 1=$ there are significant differences in the dimensional stability of the impressions after water washing or disinfecting the impressions. Concerning the antimicrobial efficiency, we hypothesized: $\mathrm{H} 0=$ there are no significant differences in the microbial reduction rates after water washing the impressions or disinfecting the impressions; $\mathrm{H} 1=$ there are significant differences in the microbial reduction rates after water washing or disinfecting the impressions.

\section{RESULTS}

The sample used for the evaluation of antimicrobial efficiency consisted on 16 participants $(75 \%$ females and $25 \%$ males) with a mean age of $23.50 \pm 0.89$ years old and a DMFT index of $1.75 \pm 1.39$. The dental impressions with no treatment (control group) had a mean microbial load of 


\section{Silicone}

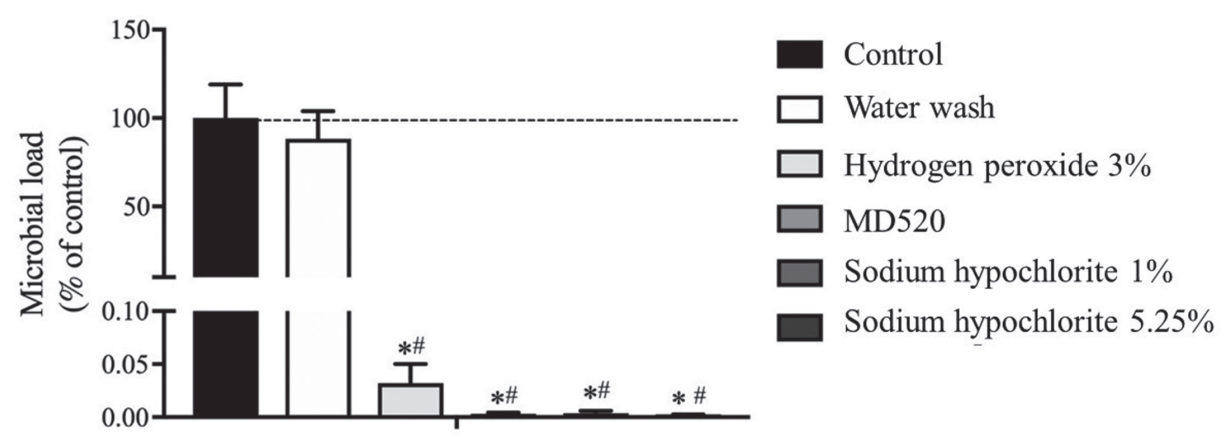

Fig. 2. Microbial adhesion on silicone impressions, after the different treatments. Bars represent mean and the error bars represent standard error of the mean. ${ }^{*} P<.001$ vs control and $\# P<.001$ vs water wash $30 \mathrm{~s}$. Both $P$ values were calculated using Kruskal-Wallis test, and the significance values were adjusted by the Bonferroni correction for multiple comparisons.

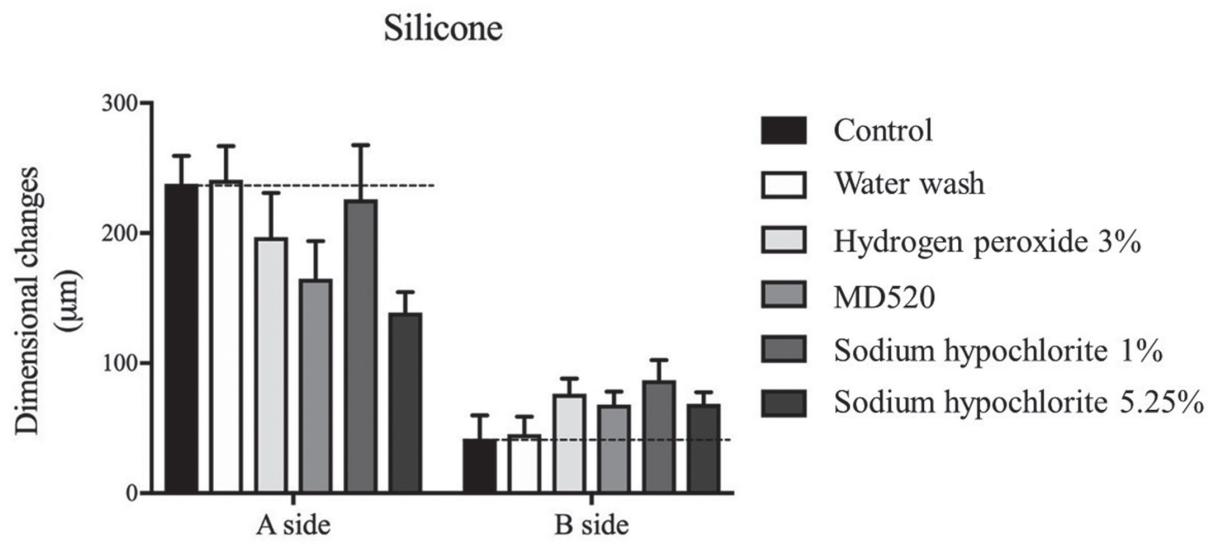

Fig. 3. Dimensional changes measured on sides A and B, after the different treatments. Bars represent mean and the error bars represent standard error of the mean.

$10.2 \times 10^{3} \pm 9.9 \times 10^{3} \mathrm{UFC} / \mathrm{mL}$ and the basal group had a mean microbial load of $0.14 \pm 0.38 \mathrm{UFC} / \mathrm{mL}$. Regarding the microbial load, a statistically significant difference was observed among the groups with no treatment, water wash, and disinfection procedure $(P<.001$, Kruskal-Wallis test with Bonferroni correction, Power analysis > 0.99, Fig. 2). Water wash reduced the microbial load by $11.7 \%$ (with no statistical difference), while all four disinfectants reduced the microbial load by more than $99.9 \%$ (please notice the different scales on Fig. 2). When comparing the microbial load within the disinfectant's groups only (without the control and water wash), a statistically significant difference was observed between hydrogen peroxide 3\% and sodium hypochlorite $5.25 \%(P=.022$, Kruskal-Wallis test with Bonferroni correction).

The results of dimensional stability evaluation are shown in Fig. 3. No significant differences were found among all the groups on the A side ( $P=.086$, Brown and Forsythe test, Power analysis $=0.65)$ or on the B side $(P=.212$, ANOVA test, Power analysis $=0.56$ ). All the values of dimensional changes calculated for side A met the ISO standard 4823: $2015,{ }^{25}$ which states that the maximum percentage of dimensional change for elastomers should not exceed 1.5\%. Moreover, although the power analysis is not strong enough to "prove" the null hypothesis, the lower and upper bound of the $95 \%$ confidence interval values fall within the $1.5 \%$ maximal dimensional change accepted by ISO standard 4823:2015. 


\section{DISCUSSION}

In dental practice, cross-infection control is of major relevance. The results of our study showed that water wash does not alter the dimensional stability of addition silicone impressions. However, it does not efficiently reduce the microbial load of the material, and therefore does not reduce the risk of cross-infection. On the other hand, addition silicone disinfection by immersion in 3\% hydrogen peroxide, MD520 (Durr), or sodium hypochlorite at 1\% and $5.25 \%$ efficiently reduces the microbial load of the impression without altering the dimensional stability significantly. So, in order to effectively reduce the cross-infection risk, addition silicone dental impression should always be disinfected after water wash.

Disinfection of dental impressions by immersion is considered one of the safest methods of disinfection, since literature states that the porosity of dental impression materials leads to the penetration of microorganisms, which disinfection by spray may not effectively destroy. ${ }^{27,28}$ Studies showed no statistical differences regarding dimensional stability, when spraying was compared to immersion as a disinfection method. ${ }^{29,30}$ A study by Marinheiro Marques et al., ${ }^{14}$ performed in Viseu (Portugal), shows that only $60.3 \%$ of dentists report disinfecting the impressions and that the majority $(51.6 \%)$ of the inquired dentists disinfect the impressions by spray. Contrarily to this investigation, a study by Almortadi and Chadwick, ${ }^{12}$ in the United Kingdom, states that the majority of dentists immerses their impressions in the disinfectant. This study also shows that dentists use sodium hypochlorite in several concentrations $(0.001 \%$, $8 \%$ and other concentrations recommended by the disinfectant's manufacturer). ${ }^{12}$ Although there are guidelines, ${ }^{10,11}$ both of these studies reflect that there is a need for more information and education regarding dental impression management.

The Australian Dental Association recommends that after water washing, the impression should be further cleaned with a diluted detergent to remove remaining microorganisms and, if desirable, chemical disinfectants..$^{10}$ The results of the present study show that direct disinfection after water wash is very efficient in reducing the microbial load and does not alter dimensional stability. In our opinion, it would be preferable to do a 2-step procedure (water wash + disinfection) than a 3 -step procedure (water wash + detergent + disinfection) because it is less time-consuming and less prone to error. In addition, two disinfection solutions usually used in dental office were tested in this study: hydrogen peroxide and sodium hypochlorite.

The microbial reduction associated with water washing was considerably lower than what some literature describes, stating that this procedure can reduce about $40 \% 0^{1,6,9}$ to $90 \%$ of the microbial load. ${ }^{31}$ Notwithstanding, some reports show that washing the impression with water does not eliminate all blood and saliva from the impression surface due to salivary mucins and salivary adhesive proteins, which may interfere with the simple washing. ${ }^{32}$ Thus, water wash alone should be avoided to achieve low risk of cross-infection. A suggestion to lower the bacterial load of the oral cavity was presented in a previous study from Dasgupta et al., ${ }^{33}$ in which the usage of preprocedural oral prophylaxis and mouth rinses was tested in 60 patients. They showed that those procedures effectively reduced the amount of oral microorganisms in hydrocolloid impressions. ${ }^{33}$

Regarding the disinfectants' efficiency on the reduction of the microbial load, the results are in accordance with the literature. According to Bustos et al. ${ }^{34}$ five to ten minutes of immersion in sodium hypochlorite at a concentration of $0.5 \%$, lower than the one we used in our study, effectively inhibited bacterial growth. Another study by Pal et al. ${ }^{18} \mathrm{dem}-$ onstrated that the immersion of polyvinyl siloxane impressions in $1 \%$ and $4 \%$ sodium hypochlorite solutions not only eliminated $100 \%$ of the bacterial load, but also did not cause deterioration in the surface of the impressions. ${ }^{18,34}$ However, in a research conducted by Jeyapalan et al., ${ }^{35}$ in which the efficiency of $1 \%$ sodium hypochlorite was compared to electrolyzed oxidizing water and $2.4 \%$ glutaraldehyde in addition silicone impressions, the results indicated that electrolyzed oxidizing water (microbial reduction rate was $100 \%)$ had a significantly higher $(P=.032)$ microbial reduction rate than sodium hypochlorite (reduction rate of $99.82 \%$ ), raising new possibilities regarding the choice of the impressions' disinfectant.

As for MD520, Giammanco et al. ${ }^{16}$ and Demajo et al..$^{27}$ showed very efficient results in the reduction of microbial contamination. Hydrogen peroxide is less explored, but a published report shows the reduction in microbial counts in condensation silicone impression. ${ }^{2}$ So, in accordance with the present results, hydrogen peroxide could be a valuable option as silicone impression disinfectant.

When the dimensional stability of the impressions was evaluated, it was concluded that dimensional changes caused by all the disinfectants were not significant when compared to the ones observed in the control and 'water washing' groups. Although the power analysis regarding dimensional stability evaluation was below 0.80 , making it difficult to prove the null hypotheses, the observed dimensional changes are not clinically relevant, since the values fall within the $1.5 \%$ maximal dimensional change accepted by ISO standard 4823:2015. Our result is corroborated by other studies such as the study by Demajo et al. ${ }^{27}$ and that by Melilli et al. ${ }^{17}$ which presented no changes or clinically acceptable changes in dimensional stability regarding disinfection by imbibition in MD520. As for 1\% and 5.25\% sodium hypochlorite, it is also well documented in previous works about its innocuous effect on addition silicone dental impressions. Queiroz et al. ${ }^{36}$ compared the effect of immersion of addition silicone impressions in 1\% sodium hypochlorite and in $0.2 \%$ paracetic acid (versus a nondisinfected control) and concluded that the disinfection did not significantly altered the impressions. Additionally, an investigation by Rentzia et al. ${ }^{1}$ studied the dimensional change in the resultant gypsum cast after the impression disinfection with $0.5 \%$ sodium hypochlorite for 30 seconds to 300 seconds, and it did not 
significantly impact either the dimensional accuracy or the surface roughness of the impressions. ${ }^{1,28}$

Immersion times may vary between studies. Thouati et al. ${ }^{37}$ describe significant dimensional changes between the unwashed control and the addition silicone impressions disinfected with $5.25 \%$ sodium hypochlorite, but the immersion time $(30 \mathrm{~min})$ was 3 times that used in the present study (10 min).

The main objective of this investigation was to evaluate the disinfection chemicals with the greatest antimicrobial efficacy and simultaneously with less effect on the dimensional stability of addition silicone impressions. In summary, all chemical disinfectants showed good results both on the dimensional stability and antimicrobial efficacy evaluation. Under these circumstances, we can state that these disinfectants could be safely recommended for the disinfection of addition silicone impressions in clinical practice.

Nowadays, other promising disinfection protocols are introduced. Disinfection with ozone gas is one of the newer options as an alternative to immersion, considering that ozone does not chemically interact with the impression material. ${ }^{38}$ Celebi et al. ${ }^{38}$ demonstrated that polyvinyl siloxane impressions exposed to gaseous ozone significantly reduced the microbial load, but with less efficacy than $0.5 \%$ sodium hypochlorite. Another alternative is disinfection by ultraviolet (UV). UV chambers are available in some dental offices and are an effective method for destroying microorganisms without the use of chemicals. ${ }^{39}$ In 2018, a study by Samra and Bhide ${ }^{28}$ showed that disinfection by UV light has no significant impact on dimensional stability of dental impressions. Another economical mean of disinfection is by autoclaving the impressions. Studies by Surendra $e t$ al..$^{40}$ and Thota $e t$ al. ${ }^{41}$ evidenced that the autoclave does not clinically affect the dimensions of the polyvinyl siloxane impressions, being a safe and potential alternative to the traditional disinfectants.

In this study, the population from whom the impressions were collected were dental students because they are a homogenous population regarding oral health and oral hygiene habits. However, the present study includes some limitations. We have evaluated a limited number of disinfectants and have not compared between disinfection by immersion to disinfection by spray. It is relevant to highlight that there's a lack of comparable and recent studies in the literature, and as many authors have pointed out, there is no consensus in the literature. Therefore, more studies are needed.

\section{CONCLUSION}

The disinfection of the addition silicone impressions should always be performed after water washing. The disinfection by immersion with MD520 (Durr), 1\% and 5.25\% sodium hypochlorite, and 3\% hydrogen peroxide showed high antimicrobial efficiency, without significant changes in threedimensional shape of addition silicone dental impressions. The less explored hydrogen peroxide could be a valuable alternative for silicone impressions disinfection.

\section{ORCID}

Maria João Azevedo bttps://orcid.org/0000-0002-9117-4684

Inês Correia https://orcid.org/0000-0002-6240-8082

Ana Portela https://orcid.org/0000-0002-2031-2197

Benedita Sampaio-Maia https://orcid.org/0000-0001-9368-8075

\section{REFERENCES}

1. Rentzia A, Coleman DC, O’Donnell MJ, Dowling AH, O'Sullivan M. Disinfection procedures: their efficacy and effect on dimensional accuracy and surface quality of an irreversible hydrocolloid impression material. J Dent 2011;39:13340.

2. Choi YR, Kim KN, Kim KM. The disinfection of impression materials by using microwave irradiation and hydrogen peroxide. J Prosthet Dent 2014;112:981-7.

3. Amin WM, Al-Ali MH, Al Tarawneh SK, Taha ST, Saleh MW, Ereifij $\mathrm{N}$. The effects of disinfectants on dimensional accuracy and surface quality of impression materials and gypsum casts. J Clin Med Res 2009;1:81-9.

4. Flanagan DA, Palenik CJ, Setcos JC, Miller CH. Antimicrobial activities of dental impression materials. Dent Mater 1998; 14:399-404.

5. Badrian H, Ghasemi E, Khalighinejad N, Hosseini N. The effect of three different disinfection materials on alginate impression by spray method. ISRN Dent 2012;2012:695151.

6. Chidambaranathan AS, Balasubramanium M. Comprehensive review and comparison of the disinfection techniques currently available in the literature. J Prosthodont 2019;28:e849e856.

7. Ganavadiya R, Chandra Shekar BR, Saxena V, Tomar P, Gupta R, Khandelwal G. Disinfecting efficacy of three chemical disinfectants on contaminated diagnostic instruments: A randomized trial. J Basic Clin Pharm 2014;5:98-104.

8. Aeran H, Sharma S, Kumar V, Gupta N. Use of clinical UV chamber to disinfect dental impressions: A comparative study. J Clin Diagn Res 2015;9:ZC67-70.

9. Correia-Sousa J, Tabaio AM, Silva A, Pereira T, Sampaio-Maia B, Vasconcelos M. The effect of water and sodium hypochlorite disinfection on alginate impressions. Rev Port Estomatol Med Dent Cir Maxilofac 2013;54:8-12.

10. Australian Dental Association (ADA)'s Guidelines for Infection Control, Third Edition. Australian Dental Association, 2015.

11. Guidelines for Infection Control in Dental Health-Care Settings. MMWR 2003;52(No RR-17): Centers for Disease Control and Prevention, 2003.

12. Almortadi N, Chadwick RG. Disinfection of dental impressions - compliance to accepted standards. Br Dent J 2010; 209:607-11.

13. Tullner JB, Commette JA, Moon PC. Linear dimensional changes in dental impressions after immersion in disinfectant solutions. J Prosthet Dent 1988;60:725-8.

14. Marinheiro Marques MC, Rebelo Amorim SC, Soares Framegas de Araújo FM, Figueiral MH, Maia Correia AR. Behaviors of dentists and dental technicians in Viseu regarding dental impressions disinfection. Rev Port Estomatol Med Dent Cir 
Maxilofac 2014;55:232-37.

15. McDonnell G, Russell AD. Antiseptics and disinfectants: activity, action, and resistance. Clin Microbiol Rev 1999;12:14779.

16. Giammanco GM, Melilli D, Rallo A, Pecorella S, Mammina C, Pizzo G. Resistance to disinfection of a polymicrobial association contaminating the surface of elastomeric dental impressions. New Microbiol 2009;32:167-72.

17. Melilli D, Rallo A, Cassaro A, Pizzo G. The effect of immersion disinfection procedures on dimensional stability of two elastomeric impression materials. J Oral Sci 2008;50:441-6.

18. Pal PK, Kamble SS, Chaurasia RR, Chaurasia VR, Tiwari S, Bansal D. Evaluation of different disinfactants on dimensional accuracy and surface quality of type IV gypsum casts retrieved from elastomeric impression materials. J Int Oral Health 2014;6:77-81.

19. Suarez F, Monje A, Galindo-Moreno P, Wang HL. Implant surface detoxification: a comprehensive review. Implant Dent 2013;22:465-73.

20. Presterl E, Suchomel M, Eder M, Reichmann S, Lassnigg A, Graninger W, Rotter M. Effects of alcohols, povidone-iodine and hydrogen peroxide on biofilms of Staphylococcus epidermidis. J Antimicrob Chemother 2007;60:417-20.

21. Faria AC, Rodrigues RC, Macedo AP, Mattos Mda G, Ribeiro RF. Accuracy of stone casts obtained by different impression materials. Braz Oral Res 2008;22:293-8.

22. Pande NA, Parkhedkar RD. An evaluation of dimensional accuracy of one-step and two-step impression technique using addition silicone impression material: an in vitro study. J Indian Prosthodont Soc 2013;13:254-9.

23. Goel K, Gupta R, Solanki J, Nayak M. A comparative study between microwave irradiation and sodium hypochlorite chemical disinfection: a prosthodontic view. J Clin Diagn Res 2014;8:ZC42-6.

24. Ismail HA, Mahross HZ, Shikho S. Evaluation of dimensional accuracy for different complete edentulous impressions immersed in different disinfectant solutions. Eur J Dent 2017; 11:242-9.

25. ISO 4823. Dentistry - Elastomeric impression materials. International Standards Organization (ISO); Geneva; Switzerland, 2015. Available at: https://www.iso.org/standard/60586.html

26. American National Standards Institute/American Dental Association. Specification No. 19: Dental elastometric impression materials.: American Dental Association 2004:5.

27. Demajo JK, Cassar V, Farrugia C, Millan-Sango D, Sammut C, Valdramidis V, Camilleri J. Effectiveness of disinfectants on antimicrobial and physical properties of dental impression materials. Int J Prosthodont 2016;29:63-7.

28. Samra RK, Bhide SV. Comparative evaluation of dimensional stability of impression materials from developing countries and developed countries after disinfection with different immersion disinfectant systems and ultraviolet chamber. Saudi Dent J 2018;30:125-41.

29. Gounder R, Vikas BV. Comparison of disinfectants by immersion and spray atomization techniques on the linear dimensional stability of different interocclusal recording materials: An in vitro study. Eur J Dent 2016;10:7-15.
30. Kronström MH, Johnson GH, Hompesch RW. Accuracy of a new ring-opening metathesis elastomeric dental impression material with spray and immersion disinfection. J Prosthet Dent 2010;103:23-30.

31. Taylor RL, Wright PS, Maryan C. Disinfection procedures: their effect on the dimensional accuracy and surface quality of irreversible hydrocolloid impression materials and gypsum casts. Dent Mater 2002;18:103-10.

32. Rowe AH, Forrest JO. Dental impressions. The probability of contamination and a method of disinfection. Br Dent J 1978; 145:184-6.

33. Dasgupta D, Sen SK, Ghosh S, Bhattacharyya J, Goel P. Effectiveness of mouthrinses and oral prophylaxis on reduction of microorganisms count in irreversible hydrocolloid impression: an in vivo study. J Indian Prosthodont Soc 2012;13: 578-86.

34. Bustos J, Herrera R, González U, Martínez A, Catalán A. Effect of inmersion desinfection with $0.5 \%$ sodium hypochlorite and 2\% glutaraldehyde on alginate and silicone: Microbiology and SEM study. Int J Odontostomat 2010;4:16977.

35. Jeyapalan V, Krishnan CS, Ramasubramanian H, Sampathkumar J, Azhagarasan NS, Krishnan M. Comparative evaluation of the antimicrobial efficacy of three immersion chemical disinfectants on clinically derived poly(vinyl siloxane) impressions. J Prosthodont 2018;27:469-75.

36. Queiroz DA, Peçanha MM, Neves AC, Frizzera F, Tonetto MR, Silva-Concílio LR. Influence of disinfection with peracetic acid and hypochlorite in dimensional alterations of casts obtained from addition silicone and polyether impressions. J Contemp Dent Pract 2013;14:1100-5.

37. Thouati A, Deveaux E, Iost A, Behin P. Dimensional stability of seven elastomeric impression materials immersed in disinfectants. J Prosthet Dent 1996;76:8-14.

38. Celebi H, Büyükerkmen EB, Torlak E. Disinfection of polyvinyl siloxane impression material by gaseous ozone. J Prosthet Dent 2018;120:138-43.

39. Aeran H, Sharma S, Kumar V, Gupta N. Use of clinical UV chamber to disinfect dental impressions: A comparative study. J Clin Diagn Res 2015;9:ZC67-70.

40. Surendra GP, Anjum A, Satish Babu CL, Shetty S. Evaluation of dimensional stability of autoclavable elastomeric impression material. J Indian Prosthodont Soc 2011;11:63-6.

41. Thota KK, Jasthi S, Ravuri R, Tella S. A comparative evaluation of the dimensional stability of three different elastomeric impression materials after autoclaving - an in vitro study. J Clin Diagn Res 2014;8:ZC48-50. 